Document downloaded from:

http://hdl.handle.net/10251/150658

This paper must be cited as:

Marco, M.; Giner Maravilla, E.; Caeiro-Rey, JR.; Miguélez, MH.; Larrainzar-Garijo, R. (2019). Numerical modelling of hip fracture patterns in human femur. Computer Methods and Programs in Biomedicine. 173:67-75. https://doi.org/10.1016/j.cmpb.2019.03.010

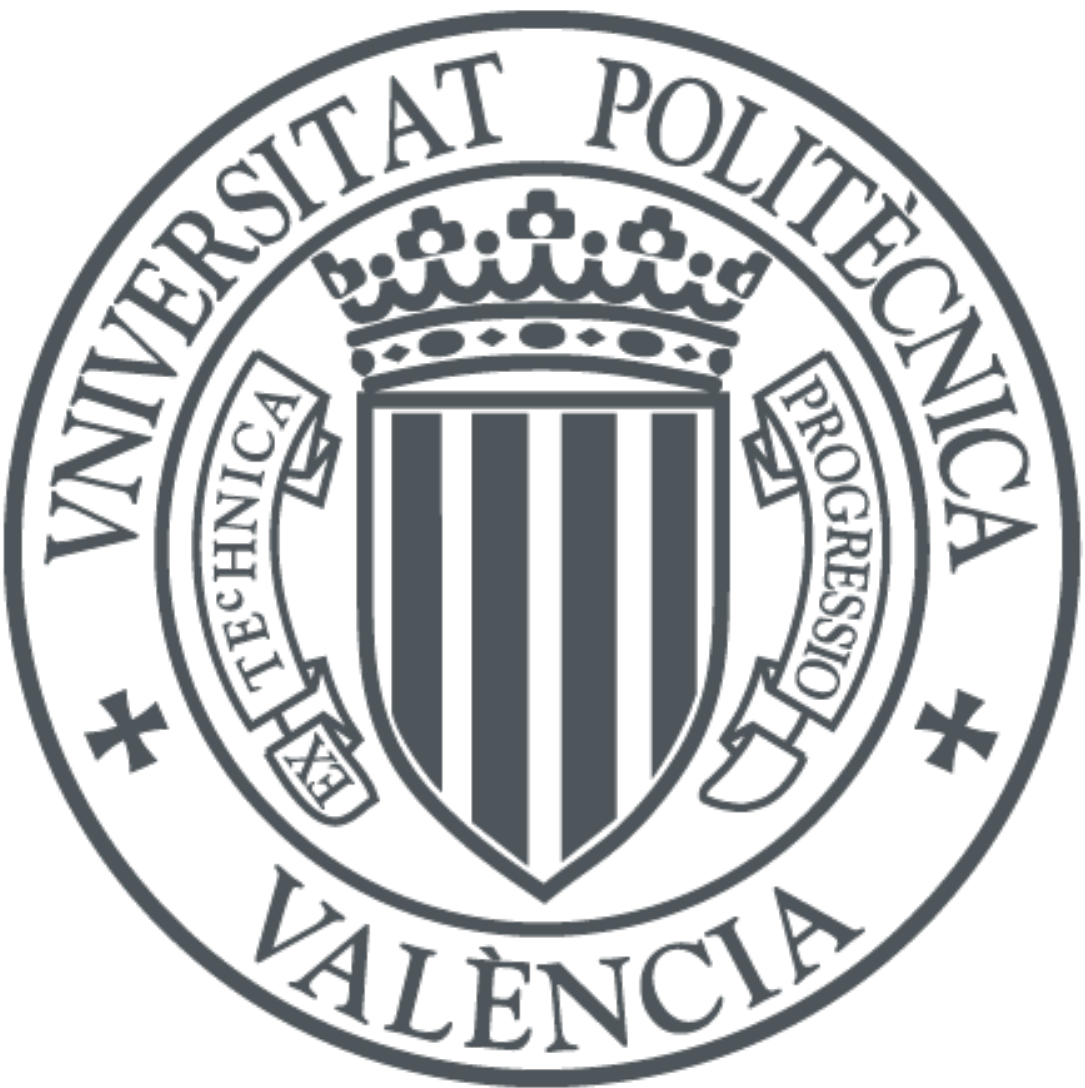

The final publication is available at

https://doi.org/10.1016/j.cmpb.2019.03.010

Copyright Elsevier

Additional Information 


\title{
Numerical modelling of hip fracture patterns in human femur
}

\author{
Miguel Marco ${ }^{1 *}$, Eugenio Giner ${ }^{2}$, José Ramón Caeiro-Rey ${ }^{3}, M^{a}$ Henar Miguélez ${ }^{1}$, Ricardo \\ Larraínzar-Garijo ${ }^{4}$

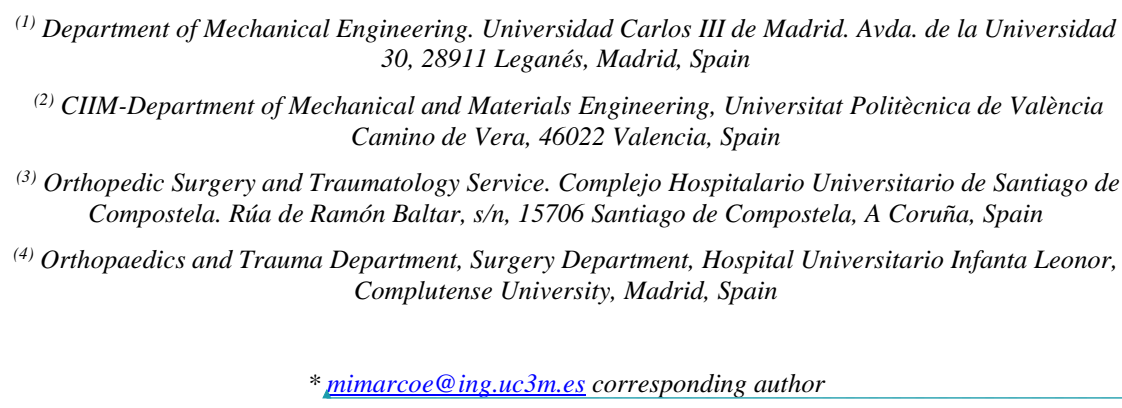

\section{Abstract}

\section{Background and Objective}

Hip fracture morphology is an important factor determining the ulterior surgical repair and treatment, because of the dependence of the treatment on fracture morphology. Although numerical modelling can be a valuable tool for fracture prediction, the simulation of femur fracture is not simple due to the complexity of bone architecture and the numerical techniques required for simulation of crack propagation. Numerical models assuming homogeneous fracture mechanical properties commonly fail in the prediction of fracture patterns. This paper focuses on the prediction of femur fracture based on the development of a finite element model able to simulate the generation of long crack paths.

\section{Methods}

The finite element model developed in this work demonstrates the capability of predicting fracture patterns under stance loading configuration, allowing the distinction between the main fracture paths: intracapsular and extracapsular fractures. It is worth noting the prediction of different fracture patterns for the same loading conditions, as observed during experimental tests. 


\section{Results and conclusions}

The internal distribution of bone mineral density and femur geometry strongly influences the femur fracture morphology and fracture load. Experimental fracture paths have been analysed by means of micro-computed tomography allowing the comparison of predicted and experimental crack surfaces, confirming the good accuracy of the numerical model.

\section{Keywords}

Fracture morphology prediction, femur fracture, intracapsular fracture, extracapsular fracture, finite element modelling.

\section{Introduction}

Human bone fracture is a common traumatism due to the aging population and the wide incidence of osteoporosis. In 2000, there were around 9 million osteoporotic fractures worldwide, from which 1.6 million affected the hip [1]. Concerning hip fracture, mortality during hospital stay is about $8 \%$ [2] and it leads to elevated costs both during the first and subsequent years [3].

The mechanical behaviour of human femur has been extensively analysed through in vitro experiments in literature. However, advances in computing and in the finite element (FE) method have also led to the development of fracture models of human femur. The synergy of these techniques allows to obtain more reliable results enhancing the knowledge about femur fracture biomechanics [4]. These models can be a useful tool for clinical assessment of hip fracture risk [5].

In the literature, the mechanical behaviour of femur has been addressed under the point of view of elastic response, fracture load and fracture morphology. In order to evaluate the femur stress state and to validate numerical models, strains on the bone surface are usually registered by means of strain gauges [6-11]. In [12] axial and torsional 
experimental tests have been carried out with human femur. New full-field measurements techniques have been also used in this area, such as digital image correlation (DIC) [1316] or infrared thermography [17]. Through the analysis of strains on the surface, it has been evidenced that the femur behaviour is linear elastic up to failure when physiological loading conditions are applied [9, 11, 15]. This assumption is also corroborated by Cristofolini et al. in [18] since the registered loss of linearity is found in the last part of the loading, i.e. close to the onset of fracture.

Many authors have measured femur fracture loads from experimental tests. The fracture load has been measured from experiments as the maximum value in the curve force vs displacement, registered when the load is applied to the femoral head. The stance loading and sideways fall conditions have been evaluated experimentally in [19]. In addition, the femur fracture load has also been estimated using finite element approaches. This requires a statement of a failure criterion: the maximum stress/strain criterion is commonly used in femur fracture modelling due to the brittle behaviour of bones [20]. The failure mode is usually tensile fracture under stance loading conditions [19] and compression failure under fall loading conditions [21]. Schileo et al. demonstrated that the failure risk and fracture location in human femurs are properly modelled using the maximum principal strain criterion [19]. It is also important to establish a criterion of failure extension indicative of fracture onset. Usually the critical level of maximum strain/stress is evaluated at the nodes within an area of interest. Some authors apply this criterion considering a percentage of damaged elements within a surface [19] or a volume [22]. Munckhof et al. [23] compared FE predictions of fracture load and common clinical fracture risk assessment techniques such as dual-energy X-ray absorptiometry (DXA). They demonstrated the potential of FE models when an adequate methodology is used. 
It is interesting to note that different failure patterns are observed even under the same loading conditions $[10,15,18,24,25]$. The fracture path morphology is not only related to loading conditions, but also, and very importantly, to bone mechanical properties and the volumetric distribution of weakened zones. These differences in fracture morphology and fracture initiation have been experimentally studied using high-speed cameras or crack-grid lines [24]. The classifications of fracture morphology are based on anatomical location or fracture direction [18]. Although different cases of fracture morphology can be identified (being the main types provided by Müller AO Classification [26] and the Pauwel's Classification [27]) a general rough criterion conditioning the further surgical treatment is to distinguish between intracapsular or extracapsular fracture [28]. Age, osteoporosis and gender have an important influence in femur fracture [1, 29], but the influence of individual morphology, local bone quality or race is not clear in the literature [30]. The clinical interest of distinguishing extra/intra capsular fracture is the avascular necrosis of the femoral head occurring in the intracapsular fracture due to the interruption of vascularization of the femoral head that depends almost exclusively on the blood supply provided by the blood vessels around the joint capsule.

On the other hand, the avascular necrosis is extremely rare in an extracapsular fracture. The surgical treatment involves the hip joint replacement by a prosthesis in an intracapsular fracture while the extracapsular fracture is commonly treated with an intramedullary nail.

The intracapsular fracture is commonly observed in stance loading conditions with crack initiation and further progression located at femoral neck [6, 11, 18, 24, 31, 32]. See for instance [18] where fracture location is clearly defined, and $80 \%$ of the specimens out of a sample of 10 femurs, initiated at the most proximal portion of the neck. 
Regarding fracture path simulation, it is worth noting that most numerical models presented in the literature only predict the first steps of fracture focusing on the value of the maximum load at crack onset [6, 33]. Simulation of long fracture paths is a difficult task (especially in 3D models). However, several numerical modelling techniques have been developed in the fracture mechanics field to simulate fracture in 3D FE models, such as the extended finite element method (XFEM), element deletion or mechanical property degradation. The XFEM has the important advantage of modelling the crack path independently of the underlying mesh. However, XFEM as available in commercial software such as Abaqus does not provide long fracture paths due to convergence problems, being the prediction restricted to the first steps of the fracture [6, 33, 34]. Another alternative is the element deletion technique, which allows to obtain longer fracture paths. However this technique can cause numerical problems related to the distortion of elements adjacent to the crack path [35]. The property degradation method consists in introducing a very substantial reduction of the Young's modulus at element level to simulate the loss of stiffness due to the crack presence. In a previous work [36], we compared different numerical approaches for femur fracture simulation, concluding that local material property degradation applied through successive analysis provided accurate results and long fracture paths. The property degradation method has been used with accurate results in fracture simulation of femur generated through bone remodelling [37] or under falling conditions [25]. Hambli [38] obtained accurate results using this technique in a simplified human femur model with homogeneous bone mineral density distribution.

This work presents a numerical approach to femur fracture modelling including the simulation of crack path progression up to complete breakage of the femur under stance 
loading conditions. This approach enables the modelling of long fracture paths and the prediction of different types of fracture: intracapsular and extracapsular.

The model has been validated through the comparison with tests on human femurs loaded up to fracture. The femur fracture paths obtained during experiments were compared with those predicted with numerical models by means of micro-computed tomography (microCT) carried out after the fracture tests. Although this technique has been rarely used for post-mortem analysis of the specimen, it was useful to demonstrate the accuracy of the numerical model. The influence of both the bone mineral density (BMD) distribution and the geometry of the femur on fracture patterns and fracture loads has also been evidenced.

\section{Materials and methods}

\subsection{Experimental work}

\subsubsection{Specimens}

Two human fresh-frozen cadaveric femurs provided by the Centre of Body Donation and Dissection Area of University Complutense of Madrid were analysed in this work. The specimens were donated according to the Spanish legislation and wrapped in salinesoaked gauze to prevent dehydration and frozen until ulterior CT-scanning. The customary protocol of conservation was followed, since it has been demonstrated that it does not alter the mechanical properties of bone tissue [39].

The donors had no reported history of muscle-skeletal diseases. The anthropometric data of the femur specimens are summarized in Table 1. 
Table 1. Details of the femurs investigated

\begin{tabular}{|l|c|c|c|c|c|}
\hline Specimen & Side & Gender & Age at death & Donor height (cm) & Donor weight (kg) \\
\hline$\# 1$ & Left & Female & 72 & 158 & 78 \\
\hline$\# 2$ & Right & Male & 73 & 170 & 88 \\
\hline
\end{tabular}

Soft tissue was removed from bone surface and both human femurs were CTscanned in order to obtain their real geometry and bone mineral density (BMD) distribution. A CT-scanner (model SIEMENS Somatom, tube voltage $120 \mathrm{kVp}$ ) with a voxel size of $0.2 \times 0.2 \times 0.2 \mathrm{~mm}^{3}$ was used with high resolution enabling an accurate characterization of the bone geometry and density distribution.

In the next subsections, a qualitative analysis of the specimens is provided, prior to explain their mechanical behaviour and the fracture morphology.

\section{Geometrical analysis}

Different geometrical parameters usually employed in the clinical field and also in similar works in the literature [40] were analysed. These parameters are the neck shaft angle (NSA), the femoral neck width (FNW), the femoral neck length (FNL) and the femoral head diameter $(D)$ (see Fig. 1). They were estimated from the femur CT-scan images using the software ScanIP (Simpleware, Exeter, UK) and their values are listed in Table

2. The centre of the femoral head was determined with a scaled-circle scaled to the approximate size of the femoral head. The, and the circle's centre was considered as that pointthe centre of the femoral head. 


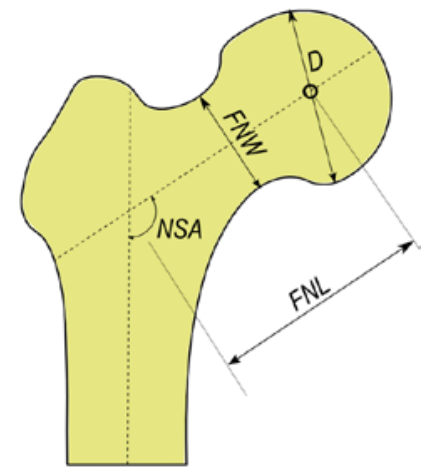

Figure 1. Parameters studied in the geometrical analysis of each femur: NSA: neck shaft angle; FNW: femoral neck width; FNL: femoral neck length and $D$ : femoral head diameter.

Table 2. Parameters measured in each femur. Dimensions and angles were calculated from the CT-scan.

\begin{tabular}{|l|c|c|}
\hline Parameter & Specimen \#1 & Specimen \#2 \\
\hline NSA $\left(^{\circ}\right)$ & 119 & 126 \\
\hline FNL $(\mathrm{mm})$ & 68 & 60 \\
\hline FNW $(\mathrm{mm})$ & 30 & 29 \\
\hline$D(\mathrm{~mm})$ & 41 & 39 \\
\hline
\end{tabular}

\section{Bone mineral density (BMD) distribution}

Using the raw DICOM files provided by the CT-scan and MATLAB (version 2016, The Mathworks, Inc. Natick, MA), the BMD distribution was plotted at the midcoronal plane of the femurs, see Fig. 2. Real values of BMD were calculated from Hounsfield Units (HU) using the relationships proposed by Morgan et al. [41]. These contour maps will be further analysed in Section 3, where results are discussed. 


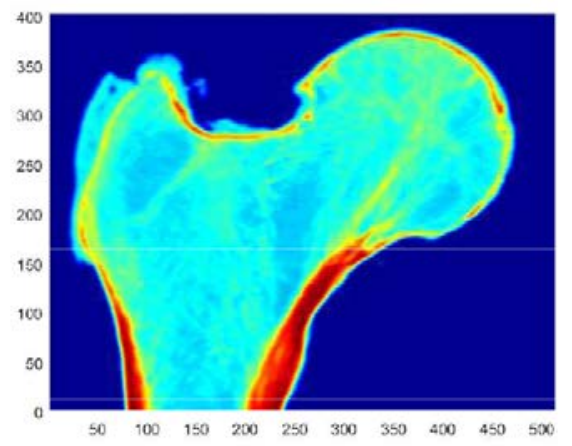

Specimen 1

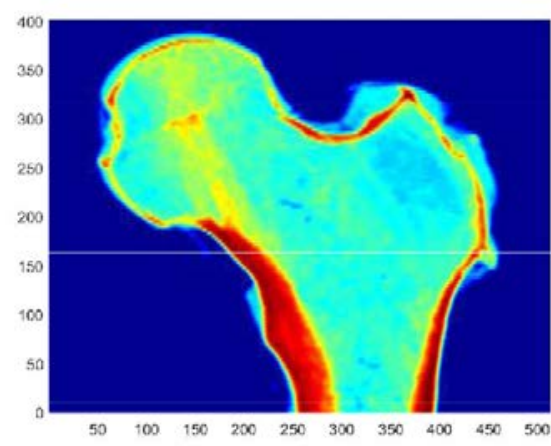

Specimen 2

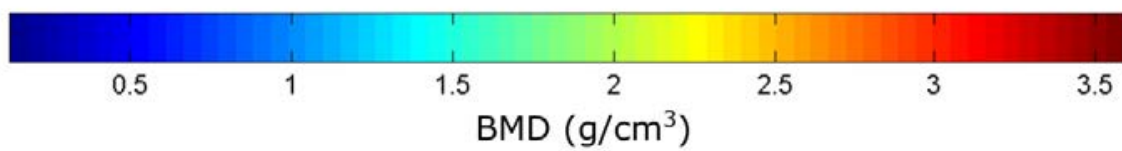

Figure 2. BMD contour colour map of both specimens analysed in this work. BMD was calculated using expressions proposed by Morgan et al. [41]. Dimensions of figures are expressed in pixels (in this work, 1 pixel = $0.2 \mathrm{~mm}$ ).

We note in passing that the BMD plots shown in Fig. 2 correspond to a sectional view (mid-coronal plane) of the whole set of CT-images. This is much more accurate and valuable that the aggregated BMD information obtained in a simple projected radiograph image, which is customary in the clinical practice. BMD lines are commonly used in medical analysis to estimate the BMD variation along a certain line in a radiograph. In this case, three different BMD lines were measured at the mid-coronal plane (see location in scheme in Fig. 3). They are located at the femoral neck length FNL (through the femoral neck, from femoral head to trochanteric area, measured at its middle line) and at the femoral neck widths FNW1 and FNW2 (orthogonal to FNL, traversing the femoral neck, and located at $40 \%$ and $50 \%$ of $F N L$ distance measured from femoral head, respectively). 
a)
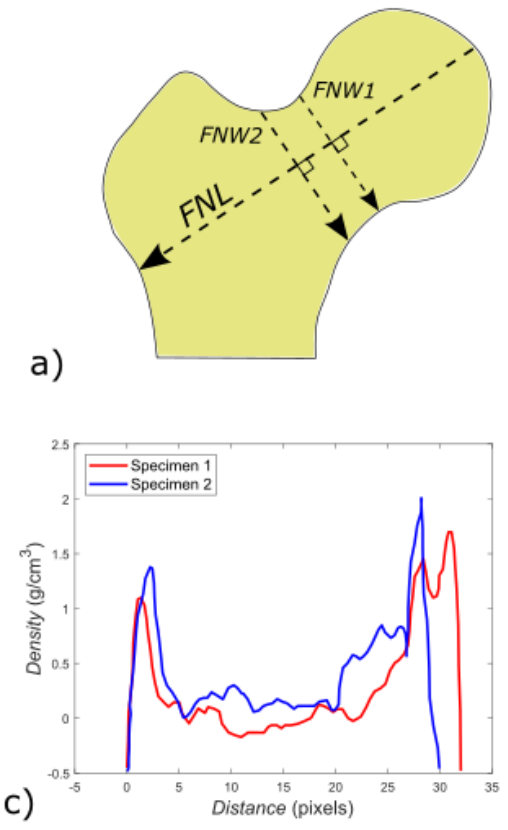

b)

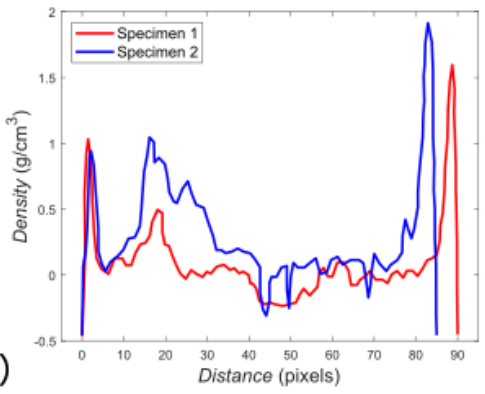

d)

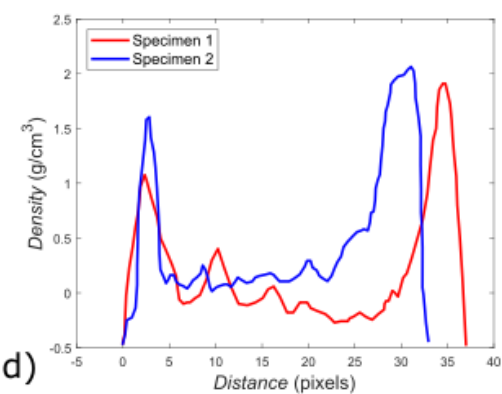

Figure 3. BMD lines in both specimens. Arrows show the direction of the measured distance. Distance is expressed in pixels. a) Scheme with the different lines measured. b) FNL line. c) FNW1 line. d) FNW2 line.

These comparative analyses will be used in the discussion presented below regarding the mechanical behaviour of the specimens.

\section{Global bone parameters}

Several parameters were calculated from the DICOM files as indicators of the femur mechanical states. In this analysis, cortical and trabecular bone are differentiated. Delimiting cortical and trabecular bone in terms of BMD is a difficult task due to the great dispersion of the threshold density values corresponding to each zone. Percentage of both tissues (cortical and trabecular) and average densities were calculated and presented in Table 3. A value of $1.9 \mathrm{~g} / \mathrm{cm}^{3}$ was set to delimit between cortical and trabecular zone, according to [42]. 
Table 3. Bone parameters of each specimen. The threshold value to delimit cortical and trabecular bone was set as $1.9 \mathrm{~g} / \mathrm{cm}^{3}$, according to [41].

\begin{tabular}{|l|c|c|}
\hline Parameter & Specimen \#1 & Specimen \#2 \\
\hline Trabecular bone (\%) & 76.5 & 66.0 \\
\hline Cortical bone (\%) & 23.5 & 34.0 \\
\hline$\rho_{\text {trab,aver }}\left(\mathrm{g} / \mathrm{cm}^{3}\right)$ & 1.49 & 1.54 \\
\hline$\rho_{\text {cort,aver }}\left(\mathrm{g} / \mathrm{cm}^{3}\right)$ & 2.46 & 2.54 \\
\hline
\end{tabular}

\subsubsection{Fracture tests}

Once the specimens were CT-scanned they were tested using a universal hydraulic testing machine (INSTRON 8801, load cell $100 \mathrm{kN}$ ). Two different tests were carried out in both specimens: a test in the elastic regime measuring strains at certain points of the bone surface and a fracture test up to breakage in order to obtain the fracture load and fracture morphology. Two strain gages were placed on the diaphysis (both in internal and external faces) and one strain gage rosette was located in the bottom zone of the femoral head (see Fig. 4a and Fig. 4b).

The loading of a stance configuration was reproduced $[6,18,33]$. The bone was aligned by rotating the long axis of the femur to $8^{\circ}$ adduction (see a scheme of the configuration in Fig. 4c) in the frontal plane by means of a rig support. This position is the usual configuration analysed in the literature because it corresponds to the most relevant loading scenario for the stance loading [18]. The load was applied on the femoral head, through a spherical-shaped punch of diameter $35 \mathrm{~mm}$. The distal diaphysis was embedded in surgical cement into the rig (Surgical Simplex P, STRYKER, Mahwah, NJ, USA). Four different loading steps were considered to analyse the femur deformation in the elastic range: 500 N, 1000 N 1500 N and 2000 N. Displacement was applied and 
controlled at the femoral head until the desired load was reached. The loading displacement rate was $0.3 \mathrm{~mm} / \mathrm{s}$, thus quasi-static conditions can be assumed.
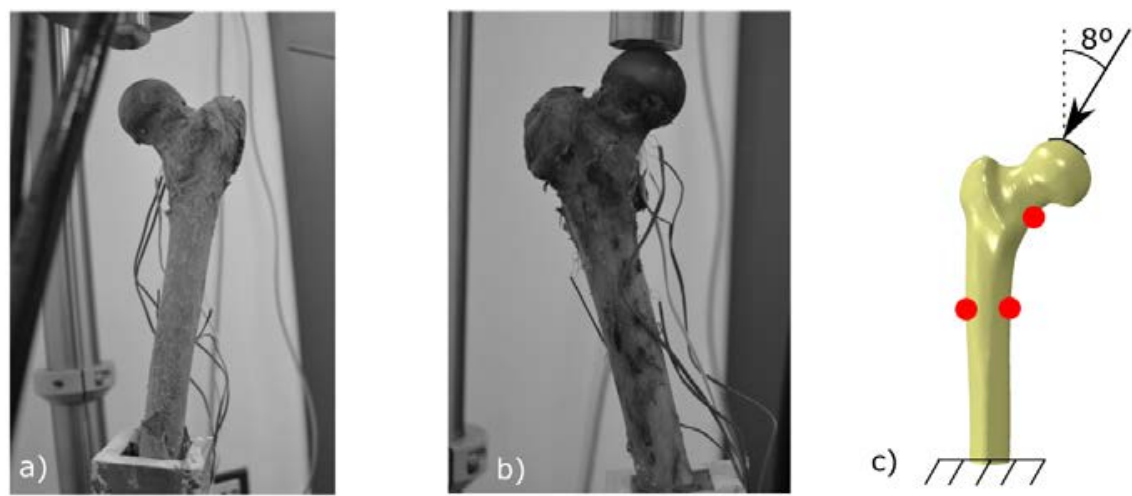

Figure 4. Mechanical testing of the specimens. a) Specimen \#1 positioned in the rig with strain gages on its surface. b) Specimen \#2 positioned in the rig with strain gages on its surface. c) Stance loading configuration analysed in this work. Red points highlight the strain gages location.

Once the elastic regime was analysed for each femur, the load on the femoral head was increased until fracture occurred. The applied force was registered and the fracture load was estimated as the maximum peak of the curve force/displacement followed by a sudden drop of the applied force.

After fracture tests, a micro-CT scanning (GE Sensing \& Inspection Technologies Phoenix X-Ray, V/Tome/X 240) was carried out in order to analyse the internal structure of the fracture path in each specimen. The resolution of the micro-CT $(63 \mu \mathrm{m})$ was enough to obtain precise segmentation of the crack surface. Tube voltage in this scan was 140 $\mathrm{kVp}$.

\subsection{Numerical modelling}

The CT images of the specimens were segmented using the software ScanIP to create the femur volumes. The segmentation of the femurs was carried out using the 
methodology proposed in [33]. With the ScanIP software it is possible to provide a distribution of mechanical properties, depending on the HU of each zone.

\section{$\underline{\text { 2.2.1 Geometry, meshing and boundary conditions }}$}

The FE mesh was also developed through the software ScanIP, and the element size was set considering a mesh sensitivity analysis performed in a previous work [33]. The element size is about $4 \mathrm{~mm}$ in the diaphysis (far region) and $2 \mathrm{~mm}$ in the proximal zone. Other authors have used a similar element size in this region [6]. The neck zone undergoes elevated stresses and usually experiences the onset and propagation of fracture. Therefore, a more refined mesh with an element size equal to $1 \mathrm{~mm}$ is required at this zone in order to obtain a sufficiently accurate solution [33].

The final mesh for both specimens consisted of about 500000 quadratic tetrahedral elements (C3D10 in Abaqus/Standard notation) and 1000000 nodes, see Fig. 5a. 


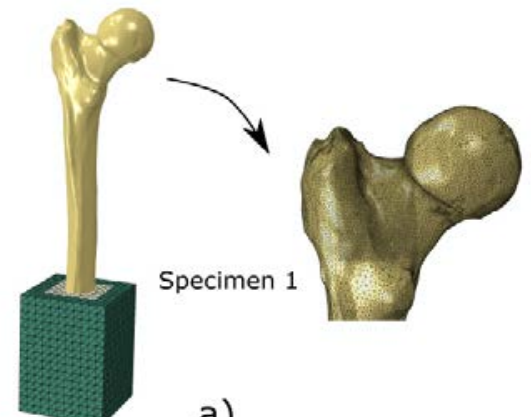

a)

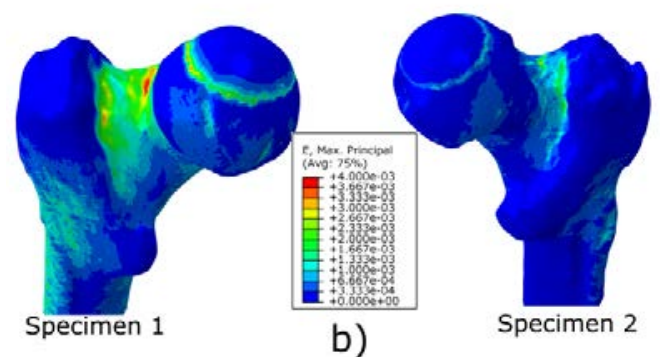

Figure 5. Numerical models of the specimens. a) Numerical model of the specimen 1 including the rig model and a detail of the refined mesh used in these models. b) Maximum principal strains under stance loading in both specimens $(2000 \mathrm{~N})$

Displacement boundary conditions and loads reproduce the conditions used in experimental tests. The testing rig was also included in the numerical models (Fig. 5a), since its influence was assessed in a previous work, affecting about $10 \%$ of the predicted strains on the bone surface [33]. Thus, the distal end of the diaphysis was attached to the testing rig through the surgical cement to simulate the experimental setup. The lowest part of the testing rig was constrained to simulate the clamping to the testing machine. The load on the femoral head was applied on a circular surface, reproducing the loading region of the experimental tests. Fig. 5b shows a plot of the principal strain contours when a load of $2000 \mathrm{~N}$ is applied under stance loading conditions. 


\section{$\underline{\text { 2.2.2 Mechanical properties: constitutive model and failure criterion }}$}

The mechanical properties of bone considered in the numerical model are related to the HU obtained in the CT-scans. The mechanical properties of the rig (aluminium alloy 7075-T6) are $E_{\mathrm{al}}=70 \mathrm{GPa}, v_{\mathrm{al}}=0.3$ and the mechanical properties of the surgical cement are $E_{\text {cem }}=2400 \mathrm{MPa}$ and $v_{\text {cem }}=0.3$, following [43].

Bone can be considered an elastic quasi-brittle material [44, 45]. Human femur presents a linear elastic behaviour up to failure $[9,11,15,18]$ and the fracture propagates in few milliseconds [11, 15, 19, 24], with little signs of permanent deformation [11]. The elevated scanner resolution allows the accurate assignment of distributed mechanical properties and thus local variations can be simulated in the numerical model. Up to 15 different zones have been generated in each specimen, corresponding to 15 levels of BMD, as in other similar works [6].

There is a wide dispersion of mechanical properties of human femur in the literature due to the dependency on age, gender, disease, nutrition or other factors [20, 29]. Eqs. 13 show the relationships between HU- $\rho$ and $\rho-E$ [46]. The Young's modulus was obtained from a density-based power law regression (Eq. 4) for the femoral neck [41], giving accurate predictions for human femur [47]

$$
\begin{gathered}
\rho_{\mathrm{QCT}}\left(\mathrm{g} / \mathrm{cm}^{3}\right)=0.00079114 \cdot H U-0.00382144 \\
\rho_{\mathrm{ash}}\left(\mathrm{g} / \mathrm{cm}^{3}\right)=0.877 \cdot \rho_{\mathrm{QCT}}+0.0789 \\
\rho_{\mathrm{app}}\left(\mathrm{g} / \mathrm{cm}^{3}\right)=\rho_{\mathrm{ash}} / 0.6 \\
E(\mathrm{MPa})=6850 \cdot \rho_{\mathrm{app}}^{1.49}
\end{gathered}
$$

where $\rho_{\mathrm{QCT}}$ is the radiological density, $\rho_{\text {ash }}$ is the ash density and $\rho_{\text {app }}$ is the apparent density and $E$ is the local Young's modulus. 
Human femur is usually modelled as a heterogeneous material, using specimenspecific models, generated from a CT-scan. Despite its heterogeneity in terms of elastic properties, the strength limit is often considered uniform over the whole femur volume. Some works consider a unique parameter to establish the fracture initiation, without taking into account the different zones in human femur (trabecular or cortical bone) and the loading state (tension or compression) [6]. Others include asymmetry in the tensile/compressive limits [10, 19, 21, 48]. Only few works consider non uniform strength limits depending on BMD [25, 35, 49]. The inclusion of a BMD dependent criterion allows the development of true specimen-specific numerical models that take into account the actual state of each specimen. The importance of considering different strength limits to model fracture in a heterogeneous material, such as bone, has been emphasized in [50].

The numerical models have been divided into fifteen zones for the assignment of different mechanical properties together with fifteen different strength limits for the failure criterion. The maximum stress criterion (Rankine criterion) is used, since this criterion was initially proposed to predict failure of brittle materials and it has also been used in similar works [20]. It is assumed that failure occurs when the principal stress exceeds the ultimate strength in tension $\left(\sigma_{\text {crit }}\right)$. Compression failure is not taken into account since the human femur exhibits tensile fracture in a stance loading configuration [19]. $\sigma_{\text {crit }}$ values have been obtained from [51], where relationships between apparent density and critical stresses for different human bones are given: tibia, vertebra, femoral head and greater trochanter. The latter are the ones used in this work. Relationships for femoral head and greater trochanter were stated as $\sigma_{\text {crit,head }}=22.6 \rho_{\text {app }}{ }^{1.26}$ and $\sigma_{\text {crit,troc }}=$ $50.1 \rho_{\text {app }}^{2.04}$ [51], respectively. In this work, different $\sigma_{\text {crit }}$ for each zone were calculated through these expressions, making a distinction between femoral head and trochanteric area. The maximum principal stress at the integration points of each element was 
compared to the critical stress of the material through a USDFLD subroutine implemented in Abaqus, following this expression:

$$
f=\frac{\sigma_{\text {crit }}}{\sigma_{\text {max,ppal }}}
$$

Once the critical level of tension is reached in an element, the failure is modelled by degrading the mechanical properties in terms of Young's modulus, which is reduced to a very low value: $E=1 \mathrm{MPa}$. This degradation technique is combined with an automatized successive-analysis through a Python script interacting with Abaqus developed in a previous work [36]. Using this method, each crack increment is considered a new analysis and it is possible to predict long fracture paths, reducing convergence problems [36]. A similar procedure, although based on XFEM, has been used by the authors for long fracture paths prediction in other applications [50, 52]. With this methodology, convergence problems found using the built-in XFEM capability available in Abaqus are circumvented [6, 33]. These convergence problems restrict the simulation to short cracks when using the built-in XFEM capability in Abaqus. It is worth noting that a long fracture path is required for fracture morphology analysis, in order to distinguish between intracapsular and extracapsular fractures.

\section{Results and discussion}

\subsection{Analysis in elastic regime}

The models developed reproduce the mechanical behaviour of the human femurs and the loading path defined in the experiments. In the elastic regime, femurs were instrumented with three strain gages (2 longitudinal and a rosette), i.e. four strain measurements were available for each femur (2 longitudinal strains, 1 maximum principal strain and 1 minimum principal strain). Since the four measurements were recorded at 
four different loading steps (500 N, $1000 \mathrm{~N}, 1500 \mathrm{~N}$ and $2000 \mathrm{~N})$, a total of 16 strain data were registered for each femur to validate the corresponding numerical model. Experimental strain values were compared to the numerical results at nodes located at the equivalent position. Model predictions were correlated through Pearson's coefficient $\left(R^{2}\right)$ to the experiment data at 0.99 with a slope of the fitted line equal to 1.03 (see Fig. 6).

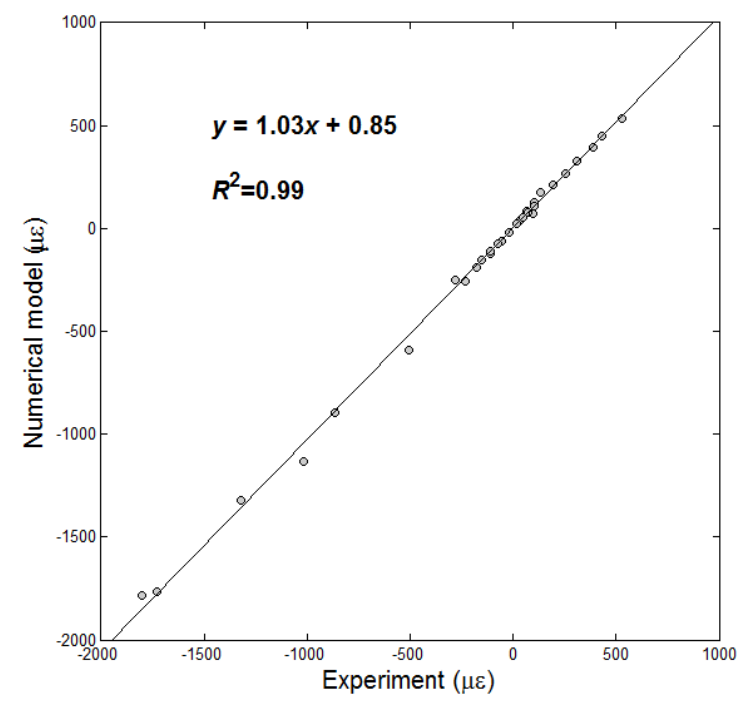

Figure 6. Comparison between predicted strains and experimental results $(\mu \varepsilon)$ for the 32 strains registered experimentally in both femurs.

The average relative error for all measurements is $6 \%$ and the normalized root mean squared error (NRMSE) is 1.4. Relative errors are lower than those found in similar works in the literature $[6,9,10,33]$. The slope is close to 1.0 , which means good accuracy in the prediction of the femur elastic behaviour, while other works underestimate the strain at the bone surface (slope $<1.0)[6,10,33]$. 


\subsection{Fracture load}

Once the model was validated within the elastic regime, the fracture load in stance loading conditions was simulated. Table 4 summarizes the results for fracture load in stance loading, including the relative errors. According to experimental results, Specimen 2 presented a fracture load greater than for Specimen 1, although both are in the range reported by other authors $[6,21]$. The greater value in Specimen 2 can be related to a higher neck shaft angle NSA and a lower femoral neck length FNL (see Fig. 1 and values of Table 2), which contribute to a stronger configuration from a structural point of view for a given load. NSA angle and the buckling ratio were also identified as important parameters in fracture risk by Aldieri et al. [53]. After analysis of the global BMD given in Fig. 2, higher values can be observed in Specimen 2, and also a greater cortical thickness, which enhance the global stiffness and strength of the femur (similar conclusions were obtained in $[53,54])$. BMD values are compared in Table 3, where Specimen 2 shows higher percentages of cortical bone and higher density values. As regards BMD lines shown in Fig. 3, in general Specimen 2 presented higher values and greater cortical thickness than Specimen 1. It is reasonable to assume that all these factors have an influence on the fracture loads obtained both experimentally and numerically. Relative errors in the fracture load prediction are 9\% and 15\% for Specimen 1 and 2, respectively. These values are acceptable compared to those found in the literature [6, 55]. The numerical fracture loads have been calculated from the FE models and the corresponding force-displacement curves. Prescribed displacements were applied to the femoral head and the reaction forces were measured at the fixed area. The fracture load was calculated as the maximum reaction force supported by the femur, while different elements were damaged by the USDFLD subroutine. The load decreases after the femur fracture. 
Table 4. Fracture load obtained experimentally and predicted by numerical models. Positive values of the relative error mean an overestimation of the value given by the model.

\begin{tabular}{|l|c|c|c|}
\hline Specimen & Experimental test & Numerical model & Relative error \\
\hline$\# 1$ & 6010 & 6572 & $9 \%$ \\
\hline$\# 2$ & 7120 & 8178 & $15 \%$ \\
\hline
\end{tabular}

It is worth noting the importance of establishing a proper methodology for fracture load estimation. In other works, a simple method based on evaluating critical stress/strain values in one single node is used in [6, 33] while an average strain tensor for each node is proposed in [21]. Also a damaged volume of elements is presented in [22], where the difficulty of estimating the volume indicative of fracture onset is discussed.

The fracture load is commonly underestimated when analysing just one single node to evaluate the femur fracture initiation $[6,21,33,55]$. Inaccurate segmentation or the presence of small soft tissue rests originate weakened zones with low density and thus poor material properties that no correspond to bone tissue. These artefacts can lead to a local erroneous estimation of the fracture load, since the failure criterion is reached earlier at these zones. In contrast with these local techniques, the analysis of the predicted displacement-force curve proposed in this work provides a global approach of the femur behaviour up to failure.

\subsection{Fracture morphology}

As explained above, fracture morphology is an important factor conditioning surgical treatment. In this work, experimental results showed an intracapsular fracture for Specimen 1 and an extracapsular fracture for Specimen 2, as shown in the micro-CT images of Fig. 7. Note that different fracture paths appear despite the same loading 
configuration is applied. Other authors have also found different fracture patterns under the same loading conditions [6, 18, 21] even for sideways falling conditions [25].

The fracture paths predicted with the numerical models are compared with those obtained from micro-CTs images presented for different view angles in Fig. 7. For Specimen 1, a femoral neck crack progressed up to the complete intracapsular fracture. Regarding Specimen 2, all fracture paths are concentrated along the intertrochanteric line, leading to extracapsular fracture, as observed experimentally. Numerically, both specimens show similar results when compared to experimental observations. This way, micro-CT allowed the comparison between experimental and numerically predicted fracture paths.

Clear differences can be observed in the fracture morphology in Fig 7 (top). Trabecular bone presents diffuse fracture with increasing distance between fracture surfaces. Cortical bone shows a more brittle behaviour with cleaner and straighter paths at fracture zone. Also differences in BMD can be appreciated through these micro-CT images. For example, a more compact trabecular structure is observed in the femoral head of Specimen 2, with a smaller trabecular distance. These observations are in line with the conclusions extracted from Fig. 2 (BMD contour maps). 


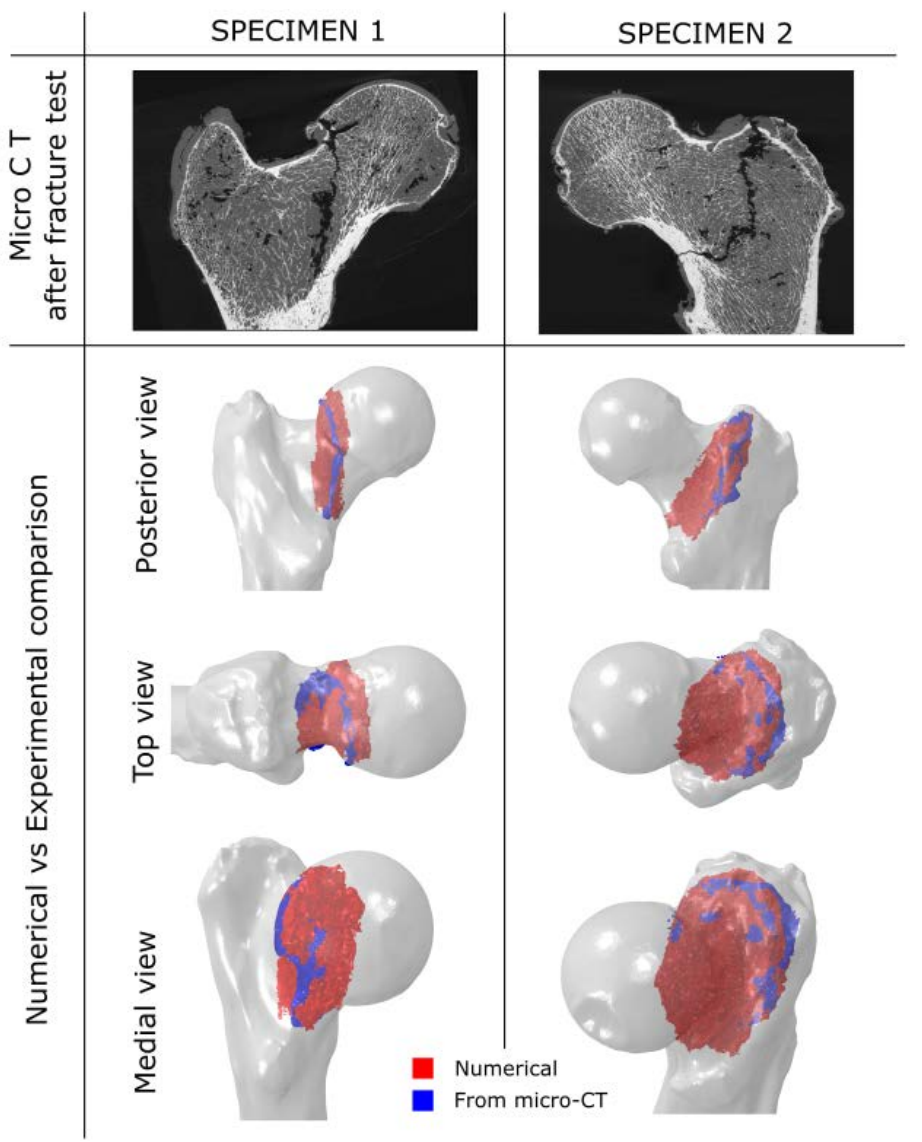

Figure 7. Fracture morphology visualization with micro-CT images (top). Specimen 1 showed an intracapsular fracture and Specimen 2 an extracapsular fracture. Comparisons between numerical and experimental fracture paths from different view angles (bottom).

The qualitative analysis presented in Section 2.1 can explain both fracture morphologies. Global BMD analysis (Fig. 2) shows a smaller upper femoral neck thickness in Specimen 1, which resulted in fracture onset at this zone. Moreover, Specimen 1 shows a weakened area close to the femoral head, located at pixel $(250,300)$ in Fig. 2, just where crack initiated. In addition, the femoral neck in Specimen 1 has lower 
BMD values as shown in Fig. 2. On the contrary, note that Specimen 2 shows a thicker cortical layer at the upper femoral neck that inhibits the fracture initiation at this zone.

Both numerical models and the CT-scans showed weakened zones presenting low BMD at the intertrochanteric line. On the other hand, the upper side of the femoral neck presented high stress values, which is indicative that this area is critical for fracture occurrence. Therefore, if the upper femoral neck presents significant cortical thickness, fracture will probably occur at the intertrochanteric line and, conversely, intracapsular fracture will occur when the upper femoral neck has a thin and weakened cortical layer.

-Although just only two specimens are studied in this work, they showed significant differences in fracture patterns allowing the comparison between extracapsular and intracapsular fractures. The Ppredicted crack paths were found to beare very similar to those extracted from the micro-CT scans demonstrating the accuracy of the numerical model to predict both fracture modes. Despite further samples should be tested in the future, Although analysing justhe -two specimens eould be are considered a limitation of the work, it was considered enough to validate the model for different fracture paths.:-

\section{Conclusions}

The procedures presented in this work allow the proper simulation of long crack paths, the prediction of fracture loads and the fracture morphology, distinguishing between intracapsular and extracapsular fractures. Two human femurs have been analysed and tested, together with the corresponding FE models. A comparative BMD analysis of both femurs has been performed in order to assess the mechanical condition of femurs, in terms of geometry of specimens and their bone mineral density. 
Numerical simulations of loading tests in the elastic regime up to fracture were in very good agreement with the strain measurements recorded experimentally, thus the models can be considered as validated. In both numerical and experimental analysis femurs showed a linear elastic behaviour up to failure, as reported by other authors. Fracture loads have been estimated from the numerical models with acceptable errors on

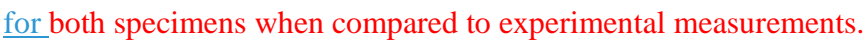

Experimental fracture paths have been observed by means of micro-CT images in order to compare them with the numerical results. The specimens presented two different types of fracture, intracapsular and extracapsular, despite both specimens were tested under the same stance loading conditions.

It has been concluded that the internal distribution of BMD strongly influences the fracture morphology. The numerical predictions are in good agreement with experimental crack paths obtained from micro-CT images. An essential step for accurate results is the assignment of mechanical properties (Young's modulus and stress limit) to each finite element in accordance to the BMD distribution.

The fracture path simulation procedure presented here allows the simulation of long fracture paths, avoiding convergence problems found with other approaches. Using the micro-CT images and the segmentation software, it is possible to check-verify the accuracy of the fracture path prediction.

The comparative analysis of certain dimensions, such as the neck shaft angle NSA and the femoral neck length $F N L$, can explain the structural behaviour of the femur in stance loading conditions. Also the BMD analysis has shown to be an essential tool when fracture morphology is studied, leading to BMD distributions in agreement with the results observed in experimental tests. As expected, high values of BMD can provide enhanced stiffness and strength. Nevertheless, local imperfections in the femur induce 
weak regions where fracture can initiate. In one of the specimens analysed in this work, the small cortical thickness in the upper side of the femoral neck and regions with low BMD are the origin of the intracapsular fracture.

\section{Acknowledgements}

The authors are indebted to University Complutense of Madrid and to the radiological team of the Hospital Universitario Infanta Leonor for supporting the experimental work on human bones included in this paper. The micro-CTs were performed in the Micro-Computed Tomography laboratory at CENIEH facilities with the collaboration of CENIEH staff. The authors gratefully acknowledge the funding support received from the Spanish Ministry of Economy and Competitiveness and the FEDER operation program for funding the projects DPI2013-46641-R, DPI2017-89197-C2-1-R, DPI2017-89197-C2-2-R, RTC-2015-3887-8 and the Generalitat Valenciana through the project Prometeo/2016/007. The authors also acknowledge the funding support received from the Fundación Española de Investigación Ósea y del Metabolismo Mineral through the research fellowship program 2018. 


\section{$\underline{\text { References }}$}

1. C. Cooper, Z.A Cole, C.R. Holroyd, S.C. Earl, N.C. Harvey, E.M Dennison, L.J. Melton, S.R. Cummings, J.A. Kanis, Secular trends in the incidence of hip and other osteoporotic fractures, Osteoporos. Int. 22 (2011) 1277-1288.

2. J. Raunest, R. Engelmann, M. Jonas, E. Derra, Morbidity and mortality in periarticular femoral fractures in advanced age. Results of a prospective study, Unfallchirurg. 104(4) (2001) 325-332.

3. C.A. Brauer, M. Coca-Perraillon, D.M. Cutler, A.B. Rosen, Incidence and Mortality of Hip Fractures in the United States, Journal of the American Medical Association 302(14) (2009) 1573-1579.

4. L. Cristofolini, E. Schileo, M. Juszczyk, F. Taddei, S. Martelli, M. Viceconti, Mechanical testing of bones: the positive synergy of finite-element models and in vitro experiments, Philos. Trans. A. Math. Phys. Eng. Sci. 368 (2010) 27252763.

5. Y. Luoz, S. Ahmedz, W.D. Leslie€, Automation of a DXA-based finite element tool for clinical assessment of hip fracture risk, Comput. Methods Programs Biomed. 155 (2018) 75-83.

6. A.A. Ali, L. Cristofolini, E. Schileo, H. Hu, F. Taddei, R. Kim, P.J. Rullkoetter, P.J. Laz, Specimen-specific modelling of hip fracture pattern and repair, J. Biomech. 47 (2014) 536-543.

7. W.S. Enns-Bray, O. Ariza, S. Gilchrist, R.P. Widmer-Soyka, P.J. Vogt, H. Palsson, S.K. Boyd, P. Guy, P.A. Cripton, S.J. Ferguson, B. Helgason, Morphology based anisotropic finite element models of the proximal femur validated with experimental data, Med. Eng. Phys. 38(11) (2016) 1339-1347. 
8. C. Falcinelli, E. Schileo, A. Pakdelc, C. Whynec, L. Cristofolini, F. Taddei, Can CT image deblurring improve finite element predictions at the proximal femur?, J. Mech. Behav. Biomed. Mater. 63 (2016) 337-351.

9. L. Grassi, E. Schileo, F. Taddei, L. Zani, M.M. Juszczyk, L. Cristofolini, M. Viceconti, Accuracy of finite element predictions in sideways load configurations for the proximal human femur, J. Biomech. 45 (2012) 394-399.

10. L. Grassi, S.P. Väänänen, M. Ristinmaa, J.S. Jurvelin, H. Isaksson, How accurately can subject-specific finite element models predict strains and strength of human femora? Investigation using full-field measurements, J. Biomech. 49 (2016) 802-806.

11. M.M. Juszczyk, L. Cristofolini, M. Viceconti, The human proximal femur behaves linearly elastic up to failure under physiological loading conditions, J. Biomech. 44 (2011) 2259-2266.

12. M.P. Gardner, A.C. Chong, A.G. Pollock, P.H. Wooley, Mechanical evaluation of large-size fourth-generation composite femur and tibia models, Ann. Biomed. Eng. 38 (2010) 613-620.

13. J.O. Buijs, D. Dragomir-Daescu, Validated finite element models of the proximal femur using two-dimensional projected geometry and bone density, Comput. Methods Programs Biomed. 104 (2011) 168-174.

14. L. Grassi, S.P. Väänänen, S.A. Yavari, H. Weinans, J.S. Jurvelin, A.A. Zadpoor, H. Isaksson, Experimental validation of finite element model for proximal composite femur using optical measurements, J. Mech. Behav. Biomed. Mater. 21 (2013) 86-94.

15. L. Grassi, S.P. Väänänen, S.A. Yavari, J.S. Jurvelin, H. Weinans, M. Ristinmaa, A.A. Zadpoor, H. Isaksson, Full-field strain measurement during mechanical 
testing of the human femur at physiologically relevant strain rates, J. Biomech. Eng. 136(11) (2014) 111010-1-8.

16. B. Helgason, S. Gilchrist, O. Ariza, J.D. Chak, G. Zheng, R.P. Widmer, S.J. Ferguson, P. Guy, P.A. Cripton, Development of a balanced experimental computational approach to understanding the mechanics of proximal femur fractures, Med. Eng. Phys. 36(6) (2014) 793-799.

17. S. Shah, H. Bougherara, E.H. Schemitsch, R. Zdero, Biomechanical stress map of an artificial femur obtained using a new infrared thermography technique validated by strain gauges, Med. Eng. Phys. 34(10) (2012) 1496-1502.

18. L. Cristofolini, M.M. Juszczyk, S. Martelli, F. Taddei, M. Viceconti, In vitro replication of spontaneous fractures of the proximal human femur, J. Biomech. 40 (2007) 2837-2845.

19. E. Schileo, F. Taddei, L. Cristofolini, M. Viceconti, Subject-specific finite element models implementing a maximum principal strain criterion are able to estimate failure risk and fracture location on human femurs tested in vitro, J. Biomech. 41 (2008) 356-367.

20. M. Doblaré, J.M. García, M.J. Gómez-Benito, Modelling bone tissue fracture and healing: a review, Eng. Fract. Mech. 71 (2004) 1809-1840.

21. E. Schileo, L. Balistreri, L. Grassi, L. Cristofolini, F. Taddei, To what extent can linear finite element models of human femora predict failure under stance and fall loading configurations, J. Biomech. 47 (2014) 3531-3538.

22. R. Bryan, P.B. Nair, M. Taylor, Use of a statistical model of the whole femur in a large scale, multi-model study of femoral neck fracture risk, J. Biomech. 42 (2009) 2171-2176. 
23. S. Munckhof, A.A. Zadpoor, How accurately can we predict the fracture load of the proximal femur using finite element models?, Clin. Biomech. 29 (2014) 373380.

24. M. Juszczyk, L. Cristofolini, M. Salva, L. Zani, E. Schileo, M. Viceconti, Accurate in vitro identification of fracture onset in bones: Failure mechanism of the proximal human femur, J. Biomech. 46(1) (2013) 158-164.

25. P. Varga, J. Schwiedrzik, P.K. Zysset, L. Fliri-Hofmann, D. Widmer, B. Gueorguiev, M. Blauth, M. Windolf, Nonlinear quasi-static finite element simulations predict in vitro strength of human proximal femora assessed in a dynamic sideways fall setup, J. Mech. Behav. Biomed. Mater. 57 (2016) 116127.

26. T.P. Ruedi, W.M. Murphy, Principles of Fracture Management. Stuttgart: Thieme Publishing Group Stuttgart (2000).

27. F. Pauwels. Der Schenkenholsbruck, em mechanisches Problem. Grundlagen des Heilungsvorganges. Prognose un kausale Therapie. Z. Orthop. Chir. 6 (1935).

28. W.P. Cooney III, R.L. Linscheid, J.H. Dbyns, Fracturas en adultos. At: Rockwood and Greens. 4ta ed. New York: Lipincott Raver Publishers (1996).

29. M. Marco, M. Rodríguez-Millán, C. Santiuste, E. Giner, M.H. Miguélez, A review on recent advances in numerical modelling of bone cutting, J. Mech. Behav. Biomed. Mater. 44 (2015) 179-201.

30. S. Nayak, D.L. Edwards, A.A. Saleh, S.L. Greenspan, Performance of risk assessment instruments for predicting osteoporotic fracture risk: a systematic review, Osteoporos Int. 25(1) (2014) 23-49.

31. J.H. Keyak, Improved prediction of proximal femoral fracture load using nonlinear finite element models, Med. Eng. Phy. 23(3) (2001) 165-173. 
32. J.H. Keyak, S.A. Rossi, K.A. Jones, C.M. Les, H.B. Skinner, Prediction of fracture location in the proximal femur using finite element models, Med. Eng. Phy. 23(9) (2001) 657-664.

33. M. Marco, E. Giner, R. Larraínzar-Garijo, J.R. Caeiro, H. Miguelez, Numerical modelling of femur fracture and experimental validation using bone simulant, Ann. Biomed. Eng. 45(10) (2017) 2395-2408.

34. C.L. Xiangyi, Q. Xiaoliang, D. Zhen-zhong, Bone fracture analysis using the extended finite element method (XFEM) with Abaqus. In The 34th annual meeting of the American society of biomechanics (2010).

35. R. Hambli, S. Allaoui, A robust 3d finite element simulation of human proximal femur progressive fracture under stance load with experimental validation, Ann. Biomed. Eng. 41(12) (2013) 2515-2527.

36. M. Marco, E. Giner, R. Larraínzar-Garijo, J.R. Caeiro, H. Miguélez, Modelling of femur fracture using finite element procedures, Eng. Fract. Mech. 196 (2018a) 157-167.

37. R. Hambli, E. Lespessailles, C. Benhamou, Integrated remodeling-to-fracture finite element model of human proximal femur behaviour, J. Mech. Behav. Biomed. Mater. 17 (2013a) 89-106.

38. R. Hambli, A quasi-brittle continuum damage finite element model of the human proximal femur based on element deletion, Med. Biol. Eng. Comput. 51(1-2) (2013b) 219-231.

39. F.G. Evans, The mechanical properties of bone, Artificial Limbs 13(1) (1969) $37-48$.

40. M.M. Machado, P.R. Fernandes, V. Zymbal, F. Baptista, Human proximal femur bone adaptation to variations in hip geometry, Bone 67 (2014) 193-199. 
41. E.F Morgan, H.H. Bayraktar, T.M. Keaveny, Trabecular bone modulus density relationships depend on anatomic site, J. Biomech. 36(7) (2003) 897-904.

42. S.C. Cowin et al., Bone mechanics handbook, Boca Raton: CRC Press (2001).

43. J.C.J. Webb, R.F. Spencer, The role of polymethylmethacrylate bone cement in modern orthopaedic surgery, Bone \& Joint J. 89(7) (2007) 851-857.

44. E. Giner, C. Arango, A. Vercher, F.J. Fuenmayor, Numerical modelling of the mechanical behaviour of an osteon with microcracks, J. Mech. Behav. Biomed. Mater. 37 (2014) 109-124.

45. I. Lapczyk, J.A. Hurtado, Progressive damage modeling in fiber-reinforced materials, Compos. Part A: Appl. Sci. Manuf. 38 (2007) 2333-2341.

46. E. Schileo, E. Dall’Ara, F. Taddei, A. Malandrino, T. Schotkamp, M. Baleani, M. Viceconti, An accurate estimation of bone density improves the accuracy of subject-specific finite element models, J. Biomech. 41 (2008b) 2483-2491.

47. E. Schileo, F. Taddei, A. Malandrino, L. Cristofolini, M. Viceconti, Subjectspecific finite element models can accurately predict strain levels in long bones, J. Biomech. 40 (2007) 2982-2989.

48. F. Taddei, I. Palmadori, W.R. Taylor, M.O. Heller, B. Bordini, A. Toni, E. Schileo, Safety factor of the proximal femur during gait: A population-based finite element study, J. Biomech. 47 (2014) 3433-3440.

49. E. Dall’Ara, R. Eastell, M. Viceconti, D. Pahr, L. Yang, Experimental Validation of DXA-based Finite Element models for prediction of femoral strength, J. Mech. Behav. Biomed. Mater. 63 (2016) 17-25.

50. M. Marco, R. Belda, M.H. Miguélez, E. Giner, A heterogeneous orientation criterion for crack modelling in cortical bone using a phantom-node approach, Finite Elem. Anal. Des. 146 (2018b) 107-117. 
51. E.F. Morgan, T.M. Keaveny, Dependence of yield strain of human trabecular bone on anatomic site, J. Biomech. 34 (2001) 569-577.

52. E. Giner, N. Sukumar, J.E. Tarancón, F.J. Fuenmayor, An Abaqus implementation of the extended finite element method, Eng. Fract. Mech. 76(3) (2009) 347-368.

53. A. Aldieri, M. Terzini, G. Osella, A.M. Priola, A. Angeli, A. Veltri, A. Audenino, C. Bignardi C, Osteoporotic hip fracture prediction: is T-score based criterion enough? A Hip Structural Analysis based model, J. Biomech. Eng. 140(11) (2018) 111004

54. H. Bahaloo, W.S. Enns-Bray, I. Fleps, O. Ariza, S. Gilchrist, R.W. Soyka, P. Guy, H. Palsson, S.J. Ferguson, P.A. Cripton, B. Helgason, On the Failure Initiation in the Proximal Human Femur Under Simulated Sideways Fall, Ann. Biomed. Eng. 46(2) (2018) 270-283.

55. O. Ariza, S. Gilchrist, R.P. Widmer, P. Guyc, S.J. Ferguson, P.A. Cripton, B. Helgason B, Comparison of explicit finite element and mechanical simulation of the proximal femur during dynamic drop-tower testing, J. Biomech. 48 (2015) 224-232. 